\title{
A working-set approach for sizing optimization of frame-structures subjected to time- dependent constraints
}

Verbart, Alexander; Stolpe, Mathias

Published in:

Structural and Multidisciplinary Optimization

Link to article, DOI:

$10.1007 / \mathrm{s} 00158-018-2063-7$

Publication date:

2018

Document Version

Peer reviewed version

Link back to DTU Orbit

Citation (APA):

Verbart, A., \& Stolpe, M. (2018). A working-set approach for sizing optimization of frame-structures subjected to time-dependent constraints. Structural and Multidisciplinary Optimization, 58(4), 1367-1382.

https://doi.org/10.1007/s00158-018-2063-7

\section{General rights}

Copyright and moral rights for the publications made accessible in the public portal are retained by the authors and/or other copyright owners and it is a condition of accessing publications that users recognise and abide by the legal requirements associated with these rights.

- Users may download and print one copy of any publication from the public portal for the purpose of private study or research.

- You may not further distribute the material or use it for any profit-making activity or commercial gain

- You may freely distribute the URL identifying the publication in the public portal

If you believe that this document breaches copyright please contact us providing details, and we will remove access to the work immediately and investigate your claim. 
See discussions, stats, and author profiles for this publication at: https://www.researchgate.net/publication/327381083

\section{A working-set approach for sizing optimization of frame-structures subjected to time-dependent constraints}

Article in Structural and Multidisciplinary Optimization · September 2018 DOI: 10.1007/500158-018-2063-7

CITATION

1

2 authors, including:

Alexander Verbart

Ramboll

8 PUBLICATIONS 70 CITATIONS

SEE PROFILE

Some of the authors of this publication are also working on these related projects:

Project Structural optimization of offshore windturbine support structures View project

Project Topology Optimization with Stress Constraints View project 


\title{
A working-set approach for sizing optimization of frame-structures subjected to time-dependent constraints
}

\author{
Alexander Verbart - Mathias Stolpe
}

Received: date / Accepted: date

\begin{abstract}
In this paper, we propose a working-set approach for sizing optimization of structures subjected to time-dependent loads. The optimization problems we consider have a very large number of constraints while relatively few design variables and degrees of freedom. Instead of solving the original problem directly, we solve a sequence of smaller sub-problems. The sub-problems consider only constraints in the working set, which is a small sub-set of all constraints. After each sub-problem, we compute all constraint function values for the current design and add critical constraints to the working set. The algorithm terminates once an optimal point to a sub-problem is found that satisfies all constraints of the original problem. We tested the approach on several reproducible problem instances and demonstrate that the approach finds optimal points to the original problem by only considering a very small fraction of all constraints. The proposed approach drastically reduces the memory storage requirements and computational expenses of the linear algebra in the optimization solver and the computational cost of the design sensitivity analysis. Consequently, the approach can efficiently solve large-scale optimization problems with several hundred millions of constraints.
\end{abstract}

Keywords Dynamic response optimization · Stress constraints · Time-dependent constraints · Sizing optimization

Alexander Verbart

DTU Wind Energy, Technical University of Denmark,

Frederiksborgvej 399, 4000 Roskilde, Denmark.

E-mail: alev@dtu.dk

Mathias Stolpe

DTU Wind Energy, Technical University of Denmark,

Frederiksborgvej 399, 4000 Roskilde, Denmark.

E-mail: matst@dtu.dk

\section{Introduction}

Design optimization in the time domain of structures subjected to time-dependent loads has been a challenge due to the large number of constraints it may involve (see, e.g., Greene and Haftka, 1991). A very large number of constraints can potentially lead to intractable optimization problems due to the demanding memory storage requirements, and the computational expensive design sensitivity analysis and linear algebra in the optimization solver. The reason for the large number of constraints is that generally the timedependent response is solved numerically using spatial and time-discretization techniques (see, e.g., Cook et al., 2002). Time-dependent constraints, for example on stresses and displacements, should be satisfied at all discrete time points. Consequently, the number of time point constraints increases proportionally to the number of time grid points considered.

Research on structural optimization subjected to time-dependent constraints dates back to the early 1970s (Fox and Kapoor, 1970). Many different approaches have been proposed to tackle the difficulties associated with the large number of constraints in dynamic response optimization problems. One approach has been to replace the original set of time-dependent constraints by a single equivalent functional constraint (see, e.g., Feng et al., 1977; Schmit and Thornton, 1968). The main idea is to remove the time-dependency of the original constraints by integrating over time. The advantage of this approach is that only one single constraint is considered, which gradient can be calculated efficiently using the adjoint variable method. A disadvantage is that information about the original constraints is lost, and that from a theoretical point of view both formulations are not equivalent since the optimality conditions are 
different (Hsieh and Arora, 1984). Moreover, numerical difficulties that lead to non-convergence can arise due to non-differentiability related to the maximum operator generally present in the integrand of the equivalent functional constraints, see for example the numerical experiments in (Hsieh and Arora, 1984).

The equivalent functional constraint described above is closely related to aggregation functions that have been used in for example stress-constrained topology optimization. Aggregation functions, such as for example the Kreisselmeier-Steinhauser function (Kreisselmeier and Steinhauser, 1979; Yang and Chen, 1996) and $P$-norm (e.g. Duysinx and Sigmund, 1998) approximate the maximum of the local constraints functions. Similar to the equivalent functional constraint, aggregation functions converts the original constraints into a single constraint. This operation greatly reduces the computational costs of the adjoint sensitivity analysis. However, the quality of the approximation of the aggregation function is generally compromised by the desired smoothness necessary to prevent numerical difficulties in gradient-based optimization. Another disadvantage is that the quality of the approximation decreases with the number of constraint functions over which is aggregated (see e.g. Verbart, 2015).

Another approach to deal with the large number of constraints has been to only consider constraints at some critical time point (see, e.g., Haftka, 1975; Haftka and Shore, 1979). The critical time points are typically time grid points where the constraint attains a value close to a (local) peak value. Several different screening strategies have been proposed to select critical time point constraints (see, e.g., Grandhi et al., 1986; Greene and Haftka, 1991; Hsieh and Arora, 1984). Since the constraint values depend on the design variables, the critical time points vary during the optimization process. Consequently, this approach can lead to numerical difficulties since the set of critical constraints changes after every iteration in the optimization process, which can lead to oscillating behavior and therefore slow convergence (Kang et al., 2006). An overview of several constraint selection strategies can be found in Kang et al. (2006).

The critical time point strategies discussed above belong to the class of active-set methods. Active-set methods have traditionally been applied to linearly constrained problems. This includes linear and convex quadratid optimization problems. The most famous active-set method is perhaps the Simplex method for linear optimization, although the term active-set method is normally not used in this context, see, e.g., Luenberger and Ye, 2008. A substantial amount of research has been placed into development, analysis, and implementation of active- set methods for convex quadratic programming (see, e.g., Bartlett and Biegler, 2006; Boland, 1996; Goldfarb and Idnani, 1983). Active-set methods are used to solve the quadratic programming sub-problems in Sequential Quadratic Programming (SQP) methods. An example of an SQP which uses an active-set method for the subproblem is SNOPT (Gill et al., 2005). Active-set methods for linearly constrained optimization problems with a general nonlinear objective function are presented in the works of Murtagh and Saunders (1978) and Forsgren and Murray (1997).

In the engineering optimization community various active-set approaches have been introduced for nonlinearly constrained problems. The examples which produce many constraints are structural topology optimization problems with local stress constraints. An activeset approach was proposed for stress constrained structural topology optimization problems (Duysinx and Bendsøe, 1998) in conjunction with the Method of Moving Asymptotes (MMA) (Svanberg, 1987). The active set dictates which constraints are included in the nonlinear and convex sub-problems in MMA. Bruggi and Duysinx (2012) also used MMA as optimization method for topology optimization with local stress constraints. For both articles, the size of the working set is allowed both to increase and decrease. The working set is defined as the set of constraints considered at the current iterate. The numerical experiments in the articles reveal that the working set is relatively large in the early iterations and reduces in size towards the final iterations. In both articles the active-set approach is intrinsically integrated into the optimization method which potentially interferes with both the practical and theoretical convergence properties since the working set changes between iterations.

Numerical difficulties associated with conventional active-set strategies include zigzagging, which refers to (infinite) oscillation between states where the same constraints are continuously added and removed to the working set (see, e.g., Luenberger and Ye, 2008). Additionally, changing the working set between iterations in the optimization solver may introduce inconsistencies when using solvers that rely on the history of the optimization process, for example, second-order methods that use a Quasi-Newton approximation of the Hessian. Another example is the update strategy of the move idimits in MMA (Svanberg, 1987).

Other researchers have applied augmented Lagrangian methods (ALMs) (Hestenes, 1969; Powell, 1969) with the aim of reducing the computational costs of the sensitivity analysis. See for example the works of Chahande and Arora (1994) and Paeng and Arora (1989) and Kim and Choi (1998) and Kim and Choi (2000). In ALMs, 
the original constrained problem is transformed into a sequence of unconstrained (or bound-constrained) optimization problems. The augmented Lagrangian functional is the Lagrangian augmented with constraint penalties. For dynamic response problems, the individual time grid point constraints are typically integrated over time when constructing the augmented Lagrangian. The gradient of the augmented Lagrangian can be computed efficiently using the adjoint variable method since it does not require the expensive calculation of the gradients of all individual constraints. However, the rate of convergence of ALMs depends on the Lagrange multiplier update procedures (Arora et al., 1991). Update procedures appropriate for dynamic response problem, i.e., that do not involve computing the gradients of the individual constraints, have only a linear convergence rate (Arora et al., 1991). Consequently, optimization methods applied to solve the augmented Lagrangian sub-problems typically converge slowly for this type of application.

Another approach that has been proposed for dynamic response problems is the Equivalent Static Loads Method (ESLM) (see, e.g., Choi and Park, 2002; Park, 2011; Park and Kang, 2003). Instead of solving the dynamic response problem directly, the ESLM solves a sequence of static response sub-problems. Each subproblem has the same objective and constraints as the original dynamic response problem, however, the response is computed using a static analysis under a set of equivalent static loads (ESLs) that are determined before each sub-problem. The ESLs are static loads that produce the same response as the dynamic analysis does for the initial design. Since the ESLM considers only static analyses during optimization of the sub-problems, this approach reduces the computational costs of the (sensitivity) analysis. It should be noted however that the number of constraints remains the same and therefore the computational costs and the memory storage requirements in the optimization solver do not change notably. The (theoretical) convergence properties of ESLM have been discussed by Stolpe, 2014 and Stolpe et al., 2017.

In this paper, we propose a working-set approach that solves a sequence of sub-problems smaller in size than the original optimization problem. The working set is the set of constraints considered in the current sub-problem and contains a relatively small number of critical constraints out of the complete set. After solving every sub-problem, a complete transient analysis is performed and new critical constraints are added to the working set. Once a constraint is added to the working set it remains there in all subsequent sub-problems. The algorithm terminates when all constraints in the complete set are satisfied after solving a sub-problem or the sub-problem is deemed either infeasible or unbounded. The proposed approach falls into the category of active-set methods, but instead of updating the working set every optimization iteration, a sequence of subproblems is solved to optimality with a fixed number of constraints. Additionally, constraints only enter the working set, which ensures that the proposed algorithm terminates in a finite number sub-problems (outer iterates).

The proposed approach is suitable for a class of optimization problems with relatively few design variables and a very large number of constraints, such as structural optimization under transient loads. In contrast to static problems, state-dependent constraints (e.g. on stress) for dynamic problems need to be satisfied at every time grid point. A specific example of a practical application is the conceptual design of offshore wind turbine support structures (Muskulus and Schafhirt, 2014). These structures are subjected to thousands of time-dependent load cases for certification purposes, and need to satisfy structural criteria on strength and fatigue. Design optimization of such applications involves stress constraints that need to be satisfied at all relevant spatial points in the structure, at all time grid points, and under all load cases. Consequently, the number of constraints becomes very large (order of billions) compared to the relatively few design variables (order of hundreds). The main hypothesis is that even though such a problem has abundantly many nonlinear constraints, the number of active constraints at any KKT point of the problem is relatively few. This hypothesis is based on the assumptions that the number of design variables is relatively small.

The approach is applied to several problem instances of sizing optimization of space-frame structures subjected to time-dependent loads and stress constraints. We consider the class of optimization problems that consider a very large number of constraints compared to a relatively small number of design variables and degrees of freedom. The number of constraints for the largest problem exceeds 300 million, whereas the number of design variables is 25 . The numerical experiments show that proposed approach can efficiently solve such problems by only considering a small fraction of the constraints. Additionally, the numerical experiments confirm the hypothesis that only a small number of constraints of the order of the number of design variables are active at a solution.

The main advantages of the proposed approach are the reduced computational time and memory requirements, which make it possible to solve large-scale structural optimization problems under many time-dependent loads. In contrast to conventional active-set strategies 
for dynamic response optimization (i.e., critical time point constraints strategies) the working set is not updated after each iteration of the optimization solver. Instead the working set is only updated after solving each sub-problem to optimality. Furthermore, the working set only increases. This strategy prevents numerical difficulties associated with conventional active set strategies such as zigzagging, and ensures termination of the algorithm in a finite number of sub-problems.

Other advantages are that the proposed working-set approach is implemented independent of the optimization method, and therefore, easy to interface with different solvers such as Sequential Quadratic Programming (SQP) (see, e.g., Boggs and Tolle, 1995; Boggs and Tolle, 2000), interior point methods (e.g., Forsgren, Gill, et al., 2002), the Method of Moving Asymptotes (MMA), (Svanberg, 1987; Svanberg, 2002), etc. The working-set approach inherits the convergence properties of the underlying optimization method. Moreover, the working set operates on the original nonlinear constraints rather than on nonlinear approximations of the constraints, such as methods based on equivalent functional constraints, and constraint aggregation; e.g., the Kreisselmeier-Steinhauser function (Kreisselmeier and Steinhauser, 1979; Yang and Chen, 1996) and P-norm (e.g. Duysinx and Sigmund, 1998). A disadvantage of the proposed approach is that the working-set selection algorithm depends on a potentially influential userdefined parameter. However, the numerical examples in this paper indicate that the algorithm is relatively insensitive to the parameter values in a certain range.

\section{Dynamic response optimization}

This section presents the problem formulation, and the problem characteristics and assumptions we consider in this article. We consider a dynamic response optimization problem with only nonlinear inequality constraints to simplify the presentation of the method. The problem in its classical nested formulation is defined as

$$
\begin{array}{cl}
\underset{\mathbf{x} \in \mathbb{R}^{n}}{\operatorname{minimize}} & f(\mathbf{x}) \\
\text { subject to } & g_{j t l}\left(\mathbf{x}, \mathbf{u}_{t l}(\mathbf{x})\right) \leq 0 \quad \forall(j, t, l) \in \mathcal{C}, \\
& \underline{\mathbf{x}} \leq \mathbf{x} \leq \overline{\mathbf{x}} .
\end{array}
$$

Here, $\mathbf{x}$ denotes the design variable vector that is assumed to be bounded from both below and above by $\underline{\mathbf{x}}$ and $\overline{\mathbf{x}}$, respectively. The objective function $f: \mathbb{R}^{n} \mapsto \mathbb{R}$ depends only on the design variables and not on the states u. The constraints $g_{j t l}: \mathbb{R}^{n} \mapsto \mathbb{R}$ are defined over the index set $\mathcal{C}=\mathcal{T} \times \mathcal{J} \times \mathcal{L}$, which is the Cartesian product of the index sets of all relevant spatial locations $(j)$, time points $(t)$ and load cases $(l)$, respectively. The constraints are the time-discretized versions of constraints that are imposed at different points in a structure. We assume that the time discretization, and spatial locations are the same for every load case.

In the numerical examples, the objective $f$ is the mass, and $g_{j t l} \leq 0$ are normal stress constraints considered at different points in a frame-structure modeled by beam finite elements. The stresses depend on the nodal displacements $\mathbf{u}_{t l} \in \mathbb{R}^{d}$ obtained from solving the linear equations of motions:

$$
\begin{aligned}
\mathbf{M}(\mathbf{x}) \ddot{\mathbf{u}}_{t l}+\mathbf{C}(\mathbf{x}) \dot{\mathbf{u}}_{t l}+\mathbf{K}(\mathbf{x}) \mathbf{u}_{t l} & =\mathbf{p}_{t l} & & \forall t \in \mathcal{T}, \forall l \in \mathcal{L}, \\
\mathbf{u}_{0 l} & =\mathbf{0} & & \forall l \in \mathcal{L} \\
\dot{\mathbf{u}}_{0 l} & =\mathbf{0} & & \forall l \in \mathcal{L} .
\end{aligned}
$$

Here, $\mathbf{M}(\mathbf{x}) \in \mathbb{R}^{d \times d}, \mathbf{C}(\mathbf{x}) \in \mathbb{R}^{d \times d}$, and $\mathbf{K}(\mathbf{x}) \in \mathbb{R}^{d \times d}$ are the mass, damping, and stiffness matrix, respectively. The load vector $\mathbf{p}_{t l} \in \mathbb{R}^{d}$ denotes load case $l$ at time $t$. The overdots represent time derivatives and $\mathbf{u}_{0 l}$ and $\dot{\mathbf{u}}_{0 l}$ are the initial conditions for each load case. The initial conditions are assumed to be design-independent and at rest for each load case. The system of equations (1) can be solved using time integration schemes. In this paper we use Newmark average acceleration which is an unconditionally stable implicit scheme generally used for structural dynamics and loads that vary more slowly than in wave propagation problems (see, e.g., Cook et al., 2002).

We assume that $\mathbf{M}(\mathbf{x}), \mathbf{C}(\mathbf{x})$, and $\mathbf{K}(\mathbf{x})$ are (i) positive definite for all designs that satisfy the variable bounds and (ii) continuously differentiable with respect to the design variables. These assumptions ensure that the displacements obtained from solving (1) are unique and continuously differentiable, see, e.g., Choi and Kim, 2006. Finally, the class of problems we consider in this paper are problems in which the number of constraint is much larger than the number of design variables and degrees of freedom of the structural model; for example, structural optimization of offshore support structures for wind turbines (see, e.g., the review article of Muskulus and Schafhirt, 2014).

\section{Proposed working-set approach}

\subsection{Relaxed optimization sub-problem}

Instead of directly solving the original problem $(\mathcal{G})$, we solve a sequence of relaxed sub-problems with a smaller 
subset of constraints, defined as

$$
\begin{array}{ll}
\underset{\mathbf{x} \in \mathbb{R}^{n}}{\operatorname{minimize}} & f(\mathbf{x}) \\
\text { subject to } & g_{j t l}\left(\mathbf{x}, \mathbf{u}_{t l}(\mathbf{x})\right) \leq 0 \quad \forall(j, t, l) \in \mathcal{W}^{k}, \\
& \underline{\mathbf{x}} \leq \mathbf{x} \leq \overline{\mathbf{x}} .
\end{array}
$$

Here, $\mathcal{W}^{k} \subseteq \mathcal{C}$ is the working set at the $k$ th sub-problem, which is a subset of the complete set and typically $\left|\mathcal{W}^{k}\right| \ll|\mathcal{C}|$. New constraints are added to the working set after solving each sub-problem.

Remark 1 Once a constraint is added to the working set it remains in the working set of all subsequent subproblems, so that $\mathcal{W}^{k-1} \subset \mathcal{W}^{k} \subset \ldots \subset \mathcal{C}$.

\subsection{Working set update strategy}

The working set is the index set of all constraints considered at a sub-problem and is updated based on the constraint function values at $\hat{\mathbf{x}}^{k-1}$, which is a KKT point to the last sub-problem. In the first sub-problem it is initialized as $\hat{\mathbf{x}}^{k-1}=\mathbf{x}_{0}$. First, a full analysis following (1) is performed for this design point to calculate the constraint function values, defined as

$g_{j t l}^{k}=g_{j t l}\left(\hat{\mathbf{x}}^{k-1}\right), \quad \forall(j, t, l) \in \mathcal{C}$.

Next, we construct an index set of all critical constraints

$$
\mathcal{Q}^{k}=\left\{(j, t, l) \mid\left(g_{j t l}^{k}-g_{j t l}^{k, \max }\right) / \max \left(g_{j t l}^{k, \max }, 1\right)>-\epsilon\right\},
$$

where $g_{j t l}^{k, \max }$ denotes the maximum constraint function value, i.e., $g_{j t l}^{k, \max }=\max _{j, t, l}\left\{g_{j t l}^{k}\right\}$. Therefore, critical constraints are selected based on the heuristics that the constraints function values closest to the maximum, are also the most critical constraints in the original problem $(\mathcal{G})$. The condition is normalized with respect to the maximum constraint function value to prevent large jumps in the number of constraints added to the working set. The maximum operator is necessary to prevent diving by a number, equal to, or smaller than zero ${ }^{1}$. The small positive user-defined parameter $\epsilon$ ensures that at least one constraint is identified as critical. In the numerical examples, we investigate the effect of this parameter on the performance of the algorithm.

The working set is then defined as the set of critical constraints of all previous iterates

$\mathcal{W}^{k}=\mathcal{W}^{k-1} \cup \mathcal{Q}^{k}$,

and is updated after solving each sub-problem $\left(\mathcal{P}^{k}\right)$.

\footnotetext{
1 In practice this is only possible in the first sub-problem when the critical set is based on the initial design point, which may be a feasible design
}

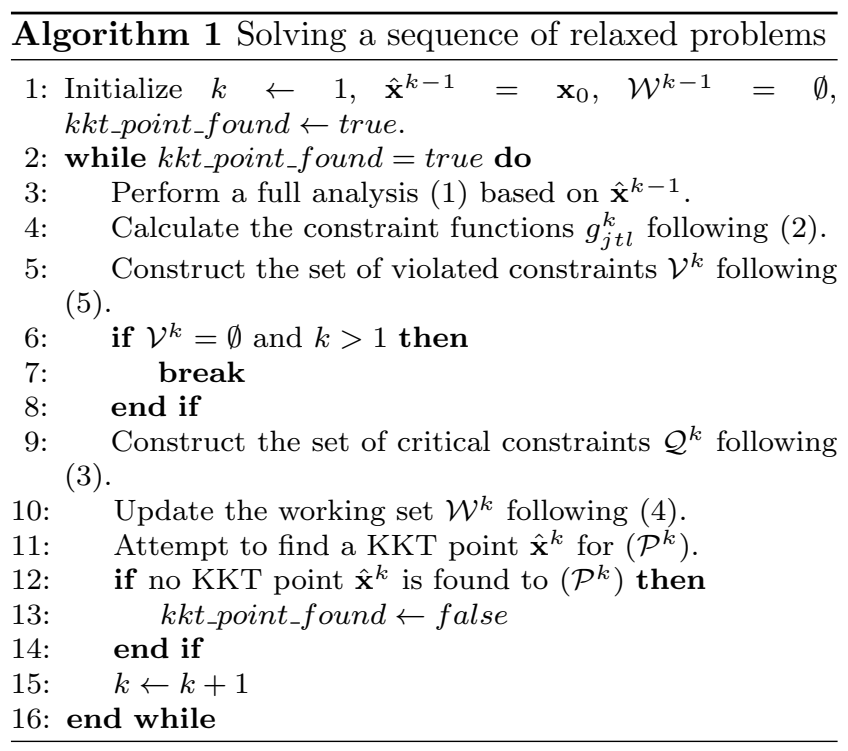

Remark 2 The definition of (3) and (4) guarantees that $\mathcal{W}^{k+1}$ is a proper superset of $\mathcal{W}^{k}$. Consequently, at least one constraint is added to the working set after each outer iterate $k$ and the approach terminates in a finite number of outer iterates.

The working set is initialized as empty $\left(\mathcal{W}^{0}=\emptyset\right)$, and therefore, the working set of the first sub-problem is $\mathcal{W}^{1}=\mathcal{Q}^{1}$. The initial set with critical constraints is based on the initial design point $\mathbf{x}_{0}$. The initial design is chosen the same for every sub-problem. Warm starting each sub-problem by setting the initial design equal to the primal solution of the last sub-problem may increase convergence, but this effect has not been studied in this paper.

\subsection{Stopping criterion}

The sequence of solving $\left(\mathcal{P}^{k}, \mathcal{P}^{k+1}, \ldots\right)$ is terminated once an optimal design point is found for which the set of violated constraints $\mathcal{V}^{k}$, which is defined as

$\mathcal{V}^{k}=\left\{(j, t, l) \mid g_{j t l}^{k}>0\right\}$,

is empty. Additionally, the algorithm terminates if for one of the sub-problems no optimal point is found that satisfies some necessary first order optimality conditions.

Remark 3 We assume that the optimization algorithm correctly reports infeasibility if sub-problem $\left(\mathcal{P}^{k}\right)$ is infeasible. This assumption is relatively strong for nonconvex problems. There exists a possibility that the optimizer deems the sub-problem infeasible even though the original problem $(\mathcal{G})$ has a non-empty feasible set. 


\subsection{Proposed algorithm}

The algorithm is described in Algorithm 1. The workingset algorithm gives possibilities for computational gains both in the structural analysis and the design sensitivity analysis. For the considered sub-problem $\left(\mathcal{P}^{k}\right)$ only load cases which have resulted in constraints included in the working set need to be considered in the analysis and the design sensitivity analysis. Additionally, it is only necessary to perform time-integration for a specific load up to the last time-step for which the constraints are in the working set. The computational improvements will thus be problem dependent.

\section{Frame structure analysis}

This section presents the space-frame analysis considered in this paper. All structures considered in this paper are modeled using solid cylindrical beam elements, but the approach can be generalized to other crosssections. The dynamic responses of the structures are obtained from solving (1) using the Newmark average acceleration method (e.g., Cook et al., 2002).

The global mass and stiffness matrix in (1) are defined as

$\mathbf{M}(\mathbf{x})=\sum_{e=1}^{n e} \mathbf{T}_{e}^{T} \mathbf{M}_{e}\left(x_{e}\right) \mathbf{T}_{e}$

and

$\mathbf{K}(\mathbf{x})=\sum_{e=1}^{n e} \mathbf{T}_{e}^{T} \mathbf{K}_{e}\left(x_{e}\right) \mathbf{T}_{e}$

respectively. Here, $\sum$ should be read as an assembly operator that assembles the ne local matrices into the global mass and stiffness matrix at the right degrees of freedom. The transformation matrix $\mathbf{T}_{e}$ transforms the local mass matrix $\mathbf{M}_{e}\left(x_{e}\right)$ and stiffness matrix $\mathbf{K}_{e}\left(x_{e}\right)$ to the global coordinate system. We consider Rayleigh damping, which is defined as $\mathbf{C}(\mathbf{x})=\alpha \mathbf{M}(\mathbf{x})+\beta \mathbf{K}(\mathbf{x})$ where $\alpha$ and $\beta$ are the damping parameters.

All structural members are modeled by Euler-Bernouilli beam finite elements with 12 degrees of freedom per element. A consistent local mass matrix is used. The local mass and stiffness matrix for this type of element can be found in Przemieniecki, 1985. The shear deformation parameter is zero to obtain the stiffness matrix for the Euler-Bernoulli beam.

The transformation matrix depends on the orientation the beam element in the global reference frame. Figure 1 shows the 3D beam element and its three nodes. A begin node $i$, end node $j$, and an auxiliary third node $k$, which is used to define its orientation

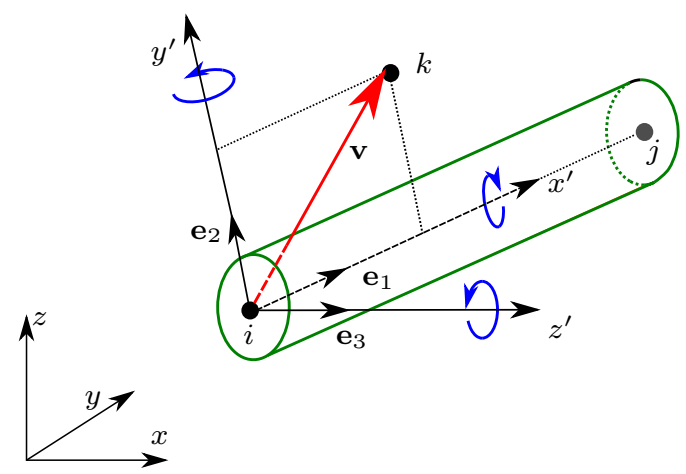

Fig. 1: Local and global coordinate systems of EulerBernoulli beam element. The begin node $i$ and end node $j$ have six degrees of freedom. Node $k$ is an orientation node.

in the global coordinate system $(x, y, z)$. The element nodal degrees of freedom in the global coordinate system are

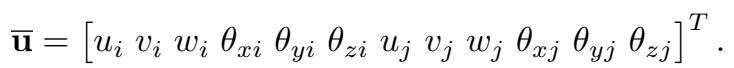

Here, $u_{i}, v_{i}$, and $w_{i}$ denote displacements of node $i$ in $x, y$, and $z$ direction, respectively. For rotations the following convention is used: $\theta_{x i}$ denotes a rotation of node $i$ around the $x$ axis. The element degrees of freedom are extracted from the global displacement field $\mathbf{u}_{t l}$ obtained from solving (1) for some load case $l$ at some time $t$. The indices $t$ and $l$ were dropped from the element nodal displacements for the sake of brevity. From now on the element index $e$ is also dropped from the transformation matrix. The element nodal degrees of freedom in the local coordinate system are then obtained by the coordinate transformation:

$\overline{\mathbf{u}}^{\prime}=\mathbf{T} \overline{\mathbf{u}}$

where the transformation matrix defined as

$\mathbf{T}=\operatorname{diag}(\Lambda, \Lambda, \Lambda, \Lambda) \quad$ where $\quad \Lambda=\left[\mathbf{e}_{1} \mathbf{e}_{2} \mathbf{e}_{3}\right]^{T}$.

The transformation matrix is a block diagonal matrix. The unit vectors $\mathbf{e}_{1}, \mathbf{e}_{2}$ and $\mathbf{e}_{3}$ are the base vectors that describe the local coordinate system. The base vector $\mathbf{e}_{1}$ is chosen along the longitudinal axis. The second and third base vectors are then determined using the vector $\mathbf{v}$ pointing from node $i$ to auxiliary node $k$ :

$\mathbf{e}_{3}=\frac{\mathbf{e}_{1} \times \mathbf{v}}{\left|\mathbf{e}_{1} \times \mathbf{v}\right|}, \quad$ and $\quad \mathbf{e}_{2}=\mathbf{e}_{3} \times \mathbf{e}_{1}$.

The normal stress in local dimensionless coordinates is defined as

$\sigma(\xi, \eta, \zeta)=E \frac{\partial \xi}{\partial x^{\prime}} \frac{\partial \mathbf{N}_{u}}{\partial \xi} \mathbf{T} \overline{\mathbf{u}}$ 
where $\xi=x^{\prime} / L, \eta=y^{\prime} / L$, and $\zeta=z^{\prime} / L$. The shape function derivative is defined as

$$
\frac{\partial \mathbf{N}_{u}{ }^{T}}{\partial \xi}=\left[\begin{array}{c}
-1 \\
(6-12 \xi) \eta \\
(6-12 \xi) \zeta \\
0 \\
L(6 \xi-4) \zeta \\
-L(6 \xi-4) \eta \\
1 \\
(12 \xi-6) \eta \\
(12 \xi-6) \zeta \\
0 \\
L(6 \xi-2) \zeta \\
-L(6 \xi-2) \eta
\end{array}\right] .
$$

See Przemieniecki (1985, p. 293) for a definition of $\mathbf{N}_{u}$.

The numerical examples consider only cylindrical beam elements for which the stress is evaluated at various points along the circumferential (at the outer diameter). The stress coordinates are chosen to be dimensionless with respect to the diameter, which is the design variable in all examples. Therefore, the stress evaluation points in a section $(\eta, \zeta)$-plane of the beam at a location $\xi$ are considered in the dimensionless polar coordinates $(\bar{r}, \phi)$, where $\bar{r}$ is a dimensionless radius defined as $\bar{r}=2 r / D$, where $r$ is the radius and $D$ is the diameter of the beam element, such that $\bar{r}=1$ corresponds with a point on the outer diameter, i.e. $r=D / 2$. The Cartesian coordinates system is then related to the polar coordinate system by

$$
\eta=\frac{D}{2 L} \bar{r} \cos (\phi), \quad \zeta=\frac{D}{2 L} \bar{r} \sin (\phi) .
$$

Substituting (7) in (6) gives

$\sigma(\xi, \bar{r}, \phi)=\frac{E}{L} \mathbf{N}_{u, \xi}(\xi, \bar{r}, \phi) \mathbf{T} \overline{\mathbf{u}}$

where we used the following notation $\mathbf{N}_{u, \xi} \equiv \frac{\partial \mathbf{N}_{u}}{\partial \zeta}$.

\section{Transient stress constraint sensitivity analysis}

In this section, we present the sensitivity analysis of the time-grid point normal stress constraints. Since we assume that the number of design variables is smaller than the number of constraints, the sensitivities are calculated by direct differentiation in contrast to the adjoint method. Furthermore, we consider so-called discreteanalytical sensitivities (see, e.g., Jensen et al., 2014; Keulen et al., 2005); i.e. we consider analytical sensitivities of the discretized governing equations in (1).

Each normal stress constraint is defined as

$g_{j t l}\left(\mathbf{x}, \mathbf{u}_{t l}(\mathbf{x})\right)=\frac{\sigma_{j t l}\left(\mathbf{x}, \mathbf{u}_{t l}(\mathbf{x})\right)}{\sigma_{\lim }}-1 \leq 0$, where $\sigma_{\lim }$ is the allowable stress and $\sigma_{j t l}$ denotes the normal stress response at a point $j$ in the structure at time step $t$ for load case $l$. To compute the gradient of the constraints, we compute the total derivative of each stress point with respect to each design variable

$\frac{\mathrm{d} \sigma_{j t l}}{\mathrm{~d} x}=\frac{\partial \sigma_{j t l}}{\partial x}+\left(\frac{\partial \sigma_{j t l}}{\partial \mathbf{u}_{t l}}\right)^{T} \frac{\mathrm{d} \mathbf{u}_{t l}}{\mathrm{~d} x}$.

The displacement sensitivities with respect to a design variable, which are defined as $\boldsymbol{\lambda}_{t l} \equiv \mathrm{d} \mathbf{u}_{t l} / \mathrm{d} x$, are obtained by solving the following system of equations

$$
\begin{aligned}
\mathbf{M} \ddot{\boldsymbol{\lambda}}_{t l}+\mathbf{C} \dot{\boldsymbol{\lambda}}_{t l}+\mathbf{K} \boldsymbol{\lambda}_{t l} & =\overline{\mathbf{p}}_{t l} & & \forall t \in \mathcal{T}, \forall l \in \mathcal{L}, \\
\mathbf{u}_{0 l}^{\prime} & =\mathbf{0} & & \forall l \in \mathcal{L}, \\
\dot{\mathbf{u}}_{0 l}^{\prime} & =\mathbf{0} & & \forall l \in \mathcal{L},
\end{aligned}
$$

where the pseudo-load is defined as

$\overline{\mathbf{p}}_{t l}=\frac{\mathrm{d} \mathbf{p}_{t l}}{\mathrm{~d} x}-\frac{\mathrm{d} \mathbf{M}}{\mathrm{d} x} \ddot{\mathbf{u}}_{t l}-\frac{\mathrm{d} \mathbf{C}}{\mathrm{d} x} \dot{\mathbf{u}}_{t l}-\frac{\mathrm{d} \mathbf{K}}{\mathrm{d} x} \mathbf{u}_{t l}$.

The system of equations in (10) is solved by the same time-discretization scheme (i.e. Newmark average acceleration) as in (1). The stress sensitivity in (9) is calculated at the finite element level. The design variables in this article are the diameters of the cylindrical beams. The stresses at all points in the element are defined as in (8). The sensitivity of the normal stress evaluated at an arbitrary location in a finite element with respect to an arbitrary diameter design variable $x$ is defined as

$$
\begin{aligned}
\frac{\mathrm{d} \sigma(\xi, \bar{r}, \phi)}{\mathrm{d} x} & =\frac{\mathrm{d} \mathbf{N}_{u, \xi}}{\mathrm{d} x}\left(\frac{\partial \sigma}{\partial \mathbf{N}_{u, \xi}}\right)^{T}+\left(\frac{\partial \sigma}{\partial \overline{\mathbf{u}}}\right)^{T} \frac{\mathrm{d} \overline{\mathbf{u}}}{\mathrm{d} x} \\
& =\frac{E}{L}\left(\delta_{x D} \frac{\mathrm{d} \mathbf{N}_{u, \xi}}{\mathrm{d} D} \overline{\mathbf{u}}^{\prime}+\mathbf{N}_{u, \xi} \mathbf{T} \frac{\mathrm{d} \overline{\mathbf{u}}}{\mathrm{d} x}\right)
\end{aligned}
$$

where $\delta_{x D}$ is Kronecker delta, which is one when the design variable $x$ is the diameter of the member where the stress sensitivity is evaluated (and zero otherwise). Notice that $\overline{\mathbf{u}}$ are the element nodal displacements under some loading condition $l$ at a certain time grid point $t$ (omitted for brevity). The total derivative of the shape function derivative is defined as

$$
\frac{\partial \mathbf{N}_{u, \xi}}{\partial D}=\frac{1}{D}\left[\begin{array}{c}
0 \\
(6-12 \xi) \eta \\
(6-12 \xi) \zeta \\
0 \\
L(6 \xi-4) \zeta \\
-L(6 \xi-4) \eta \\
0 \\
(12 \xi-6) \eta \\
(12 \xi-6) \zeta \\
0 \\
L(6 \xi-2) \zeta \\
-L(6 \xi-2) \eta
\end{array}\right] .
$$




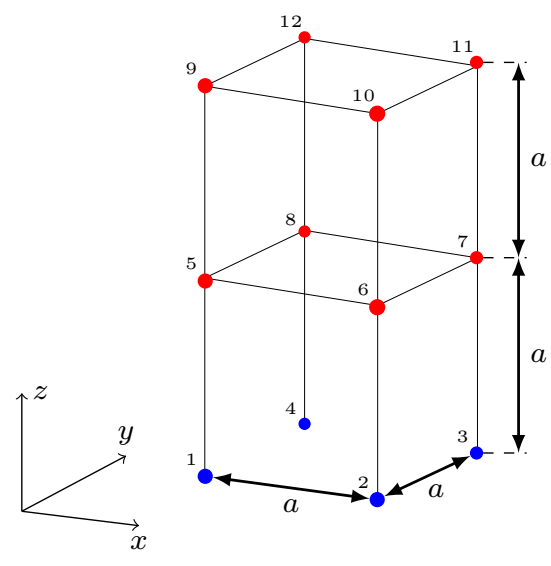

(a) 16-bar frame structure.

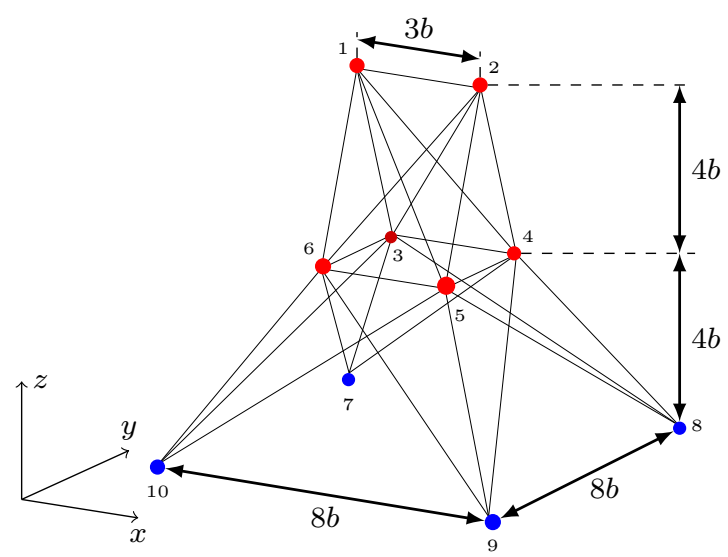

(b) 25-bar frame structure.

Fig. 2: Nodes and elements for both structural models. Both models consist of solid cylindrical beams with diameter $D=0.105 \mathrm{~m}$. The geometric parameters are $a=5 \mathrm{~m}$ and $b=1.25 \mathrm{~m}$. The initial weights are $m=1918.83 \mathrm{~kg}$ and $m=3966.25 \mathrm{~kg}$ for structure (a) and (b), respectively. The blue circles denote the fixed nodes, where all degrees of freedom are fixed. The connectivity of all finite elements are listed in Table 4.

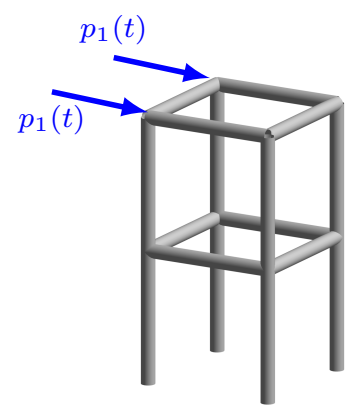

(a) Load case 1

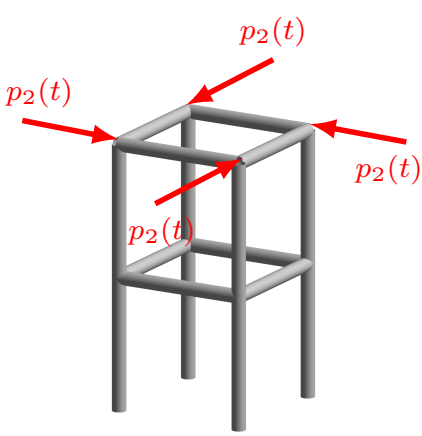

(b) Load case 2

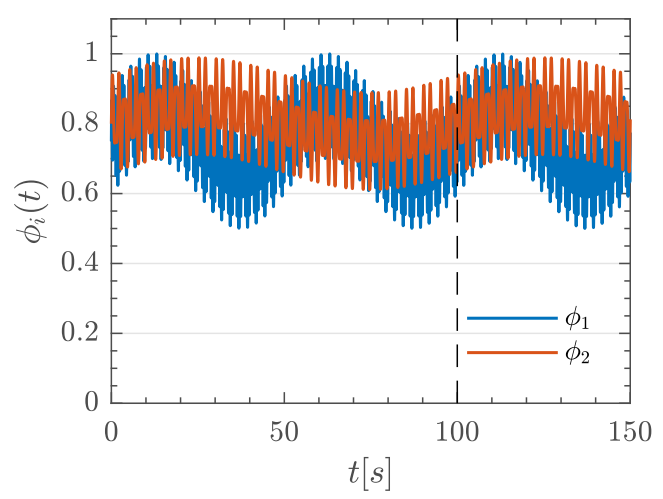

(c) Loads.

Fig. 3: Two load cases (a) and (b) on the 16-bar frame structure. The load are considered for 150s as shown in (c), but the response of the structure is only considered in the time domain to the right of the orange dashed line $[100 \mathrm{~s}, 150 \mathrm{~s}]$. The definition of both load cases is given in Table 1.

Here, $\eta(\bar{r}, \theta)$ and $\zeta(\bar{r}, \theta)$ are functions of the dimensionless polar coordinates following (7). The displacement sensitivities $\mathrm{d} \overline{\mathbf{u}} / \mathrm{d} D$ are obtained from selecting the right degrees of freedom from $\mathrm{d} \mathbf{u}_{t l} / \mathrm{d} D$, which is obtained from solving (10).

\section{Numerical examples}

Figure 2 shows the two design case considered in this paper: a 16-bar frame structure and a 25-bar frame structure. The 16-bar frame structure was used to validate the proposed approach against the complete dy- namic response problem. The 25-bar frame was solved under many dynamic loads, where we investigated the effect of the parameter value in the working set algorithm.

The 25-bar frame structure is based on the wellknown 25-bar space truss benchmark first presented by Fox and Schmit, 1966. However, instead of truss elements, the frame structures in this paper consist of beam elements of 12 degrees of freedom per element $(6$ per node). Each element consists of three nodes $i, j, k$ (see Figure 1) of which node $k$ has no degrees of freedom but is an auxiliary node used to define the orientation 
Table 1: Load case definition for the 16-bar frame.

\begin{tabular}{lccccccccc}
\hline \multicolumn{2}{l}{ The loads are defined as } \\
\multicolumn{7}{c}{$p_{i}=\bar{p}_{i} \phi_{i}(t)$, where $\phi_{i}(t)=a_{0}+\sum_{i=1}^{3} a_{i} \sin \left(2 \pi f_{i} t\right)}$. \\
\hline$p_{i}(t)$ & $\bar{p}_{i}$ & $a_{0}$ & $a_{1}$ & $a_{2}$ & $a_{3}$ & $f_{1}$ & $f_{2}$ & $f_{3}$ \\
& {$[\mathrm{~N}]$} & & & & & {$[\mathrm{Hz}]$} & {$[\mathrm{Hz}]$} & {$[\mathrm{Hz}]$} \\
\hline$p_{1}(t)$ & $5.0 \times 10^{4}$ & 0.75 & 0.10 & 0.05 & 0.1 & 2.0 & 0.4 & 0.02 \\
$p_{2}(t)$ & $5.0 \times 10^{4}$ & 0.80 & 0.05 & 0.10 & 0.05 & 0.8 & 0.6 & 0.01 \\
\hline
\end{tabular}

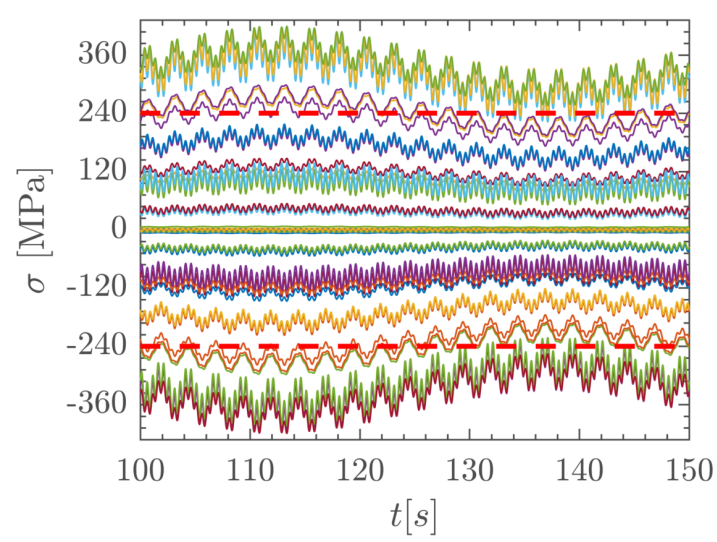

(a) Load case 1 .

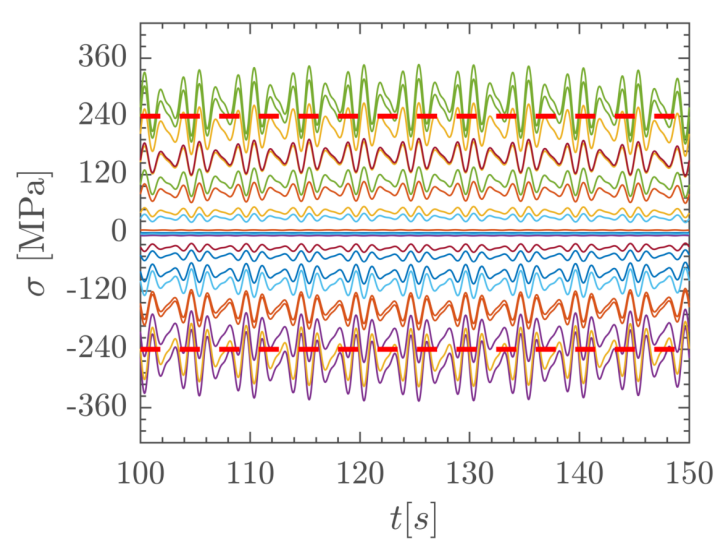

(b) Load case 2 .

Fig. 4: Stress response of the initial design for both load cases. The maximum absolute stress is $419.0908 \mathrm{MPa}$ for load case 1 and $346.5805 \mathrm{MPa}$ for load case 2 .

of the beam. Table 4 list the element connectivity for each finite element in the two design cases in Figure 2. The node numbering and coordinates are shown in Figure 2. The loads are time-dependent and defined as trigonometric functions since we focus on reproducibility rather than realistic load cases. The dynamic response for all design problems is computed using the unconditionally stable Newmark average acceleration (see, e.g., Cook et al., 2002) using the constant time step $\Delta t$. Since the time step in this scheme is unconditionally stable, the time step is chosen only based on the desired modeling accuracy and should therefore not negatively affect the proposed optimization algorithm. The solution to the dynamic equations and sensitivities is bounded for any time step. We assume that a time step of $\Delta=0.02 \mathrm{~s}$ gives a sufficiently accurate solution and that the stress values between time grid points will not deviate greatly from the computed values at the grid points. In all problem instances, the initial transient behavior of the dynamic response is neglected in the optimization problem by truncating the first 100 seconds of the computed dynamic response.

In all cases, the sizing optimization problem is to minimize the weight of the structure subjected to stress constraints, which have to be satisfied at all times. The design variables are the diameters of the solid circular beam elements. The normal stresses defined by (8) are evaluated at 12 points in each finite element. The 12 stress evaluation points are any combination of the following coordinates: $\xi=1 / 4,2 / 4,3 / 4, \bar{r}=1$, and $\phi=0, \pi / 4, \pi / 2,3 \pi / 4$.

The problem data and settings can be found in Appendix A. Table 5 lists the general problem settings that are used for all examples in this article unless stated otherwise. Table 4 lists the node connectivity for both structures. All the computations were done on an Intel Xeon E5-2680v2 ten-core CPUs, running at $2.8 \mathrm{GHz}$ with $128 \mathrm{~GB}$ RAM on a single thread using Matlab 2017a. For optimization we used the interior point solver IPOPT of Wächter and Biegler (2006) (using the linear solver MA57), which is written in $\mathrm{C}++$. The function evaluations were all implemented in Matlab and the optimization was performed by interfacing Matlab with an IPOPT binary file (i.e., using a Matlab MEX-file).

\subsection{Example 1: A 16-bar frame structure}

This section considers the 16-bar frame structure shown in Figure 2a subjected to two load cases shown in Fig- 


$$
\begin{aligned}
m & =20773.46 \\
\sigma_{1}^{\max } & =240.0000 \\
\sigma_{2}^{\max } & =240.0000
\end{aligned}
$$

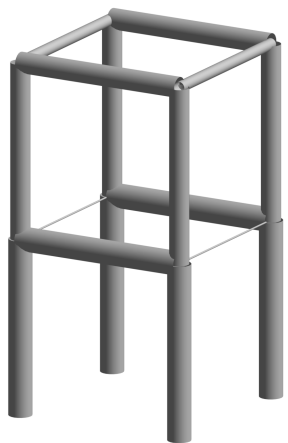

$$
\begin{aligned}
& D_{1}=0.1326 \\
& D_{2}=0.1326 \\
& D_{3}=0.1326 \\
& D_{4}=0.1326 \\
& D_{5}=0.1347 \\
& D_{6}=0.0100 \\
& D_{7}=0.1347 \\
& D_{8}=0.0100
\end{aligned}
$$$$
D_{9}=0.1106
$$$$
D_{10}=0.1106
$$$$
D_{11}=0.1106
$$$$
D_{12}=0.1105
$$$$
D_{13}=0.1165
$$$$
D_{14}=0.0639
$$

\begin{tabular}{|c|c|c|c|c|c|c|c|}
\hline \multirow[t]{2}{*}{ Method } & \multirow[t]{2}{*}{$\begin{array}{l}\text { \# sub- } \\
\text { problems }\end{array}$} & \multicolumn{2}{|c|}{ CPU time $[\mathrm{s}]$} & \multirow[t]{2}{*}{ \# iterations } & \multicolumn{2}{|c|}{ \# constraints } & \multirow[t]{2}{*}{$\begin{array}{c}\text { Memory storage } \\
\text { Jacobian }[\mathrm{Mb}]\end{array}$} \\
\hline & & Func Eval & IPOPT & & all & active & \\
\hline Complete set & 1 & 676.62 & 235.59 & 77 & 960384 & 13 & 122.93 \\
\hline Working set & 8 & 439.00 & 1.43 & 189 & 2128 & 13 & 0.27 \\
\hline
\end{tabular}$$
D_{15}=0.1165
$$$$
D_{16}=0.0638
$$

(a) Optimized design.

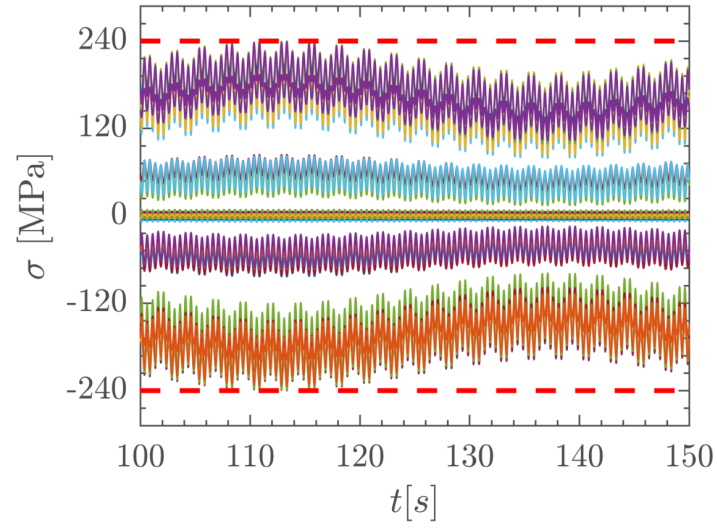

(b) Load case 1 .

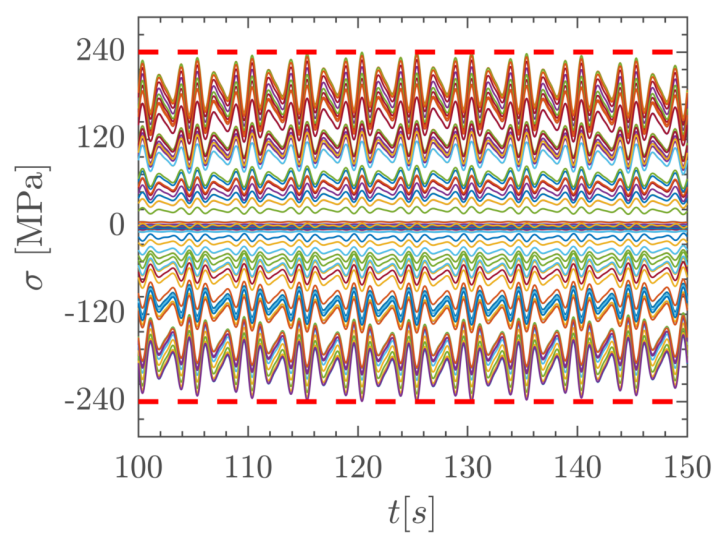

(c) Load case 2 .

Fig. 5: The optimized design (a) and the associated stress responses for all stress points in the structure for load case 1 (a) and load case 2 (b). The diameters in (a) are scaled up five times for the sake of clarity.

Table 2: Data for example 1: The 16-bar frame

ure 3. The structure is fixed at the four bottom nodes, which results in 48 free degrees of freedom. Both load cases are 150 seconds time series. The time domain of optimization is $[100,150]$ seconds; i.e. we neglect the stress responses during the first 100 seconds. The normal stress is considered in 12 spatial points per beam element, which amounts to 192 spatial stress evaluation points. The total number of stress constraint in the complete set is almost one million (960384). This number comes from multiplying the 192 spatial points, the 2501 time grid points $(\Delta t=0.02 \mathrm{~s}$ in $[100 \mathrm{~s}, 150 \mathrm{~s}])$, and the two load cases. The initial diameters are $D_{e}=$
$0.105 \mathrm{~m}$ for all $e$. Figure 4 shows the stress responses of the initial design for all 192 spatial point over time under both load cases. Notice that the initial design is infeasible since the allowable stress represented by the red dashed line is violated at all time points for both load cases.

First, we solved the original dynamic response problem considering all stress constraints. Figure 5 shows the optimized design and the associated stress responses. It can be seen that this design satisfies all stress constraints at all time points. Next, we solved the problem by the proposed working-set approach using the 


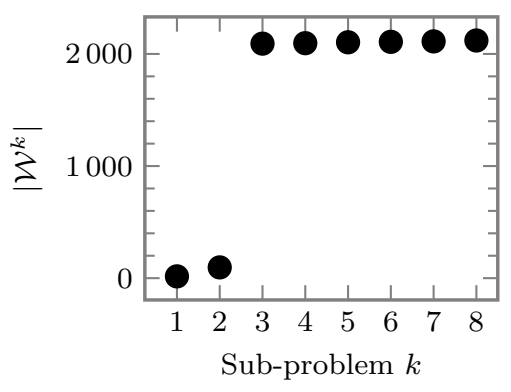

(a) Working set.

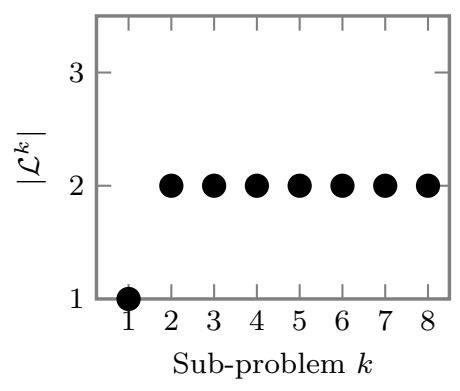

(b) Critical loads.

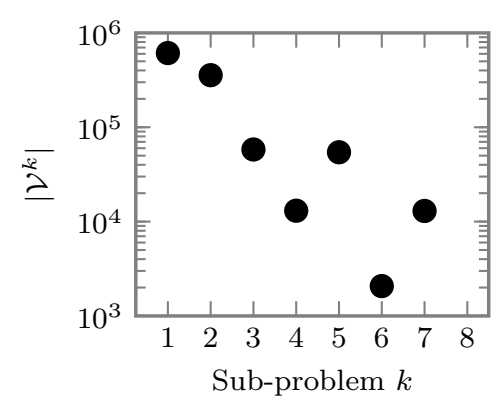

(c) Violated constraints.

Fig. 6: For each sub-problem $k$, the number of constraints in the working set $\left|\mathcal{W}^{k}\right|$ (a), the number of critical load cases $\left|\mathcal{L}^{k}\right|$, and the number of violated constraints after solving each sub-problem $\left|\mathcal{V}^{k}\right|$. Since the number of violated constraints converges to zero, the last data point is not visible shown on the logarithmic axis.

parameter value $\epsilon=10^{-3}$. The algorithm converged to the same optimized design in this particular case. Notice that both approaches could have very well converged to different (local) optima since the problem is non-convex.

Table 2 lists the results data for both approaches. In this case, when considering the complete set only 13 out of 960384 constraints are active at the optimal design point; i.e. only $\sim 0.001 \%$ of all constraints are design-driving, which is of the order of the number of design variables. The working-set approach converged after solving seven sub-problems to the same optimized design. At convergence, the working set contains only 2128 constraint indices. In this case, $0.61 \%$ of the considered constraints are active (i.e. design-driving) at the optimal design point, which is $\sim 450$ times more than when considering the complete set.

One of the main motivations of the working-set approach is to reduce memory requirements. We assume that storing the dense Jacobian dominates the memory requirements for the problems we consider. The memory storage requirements of the Jacobian is proportional to the number of constraints. Since the largest sub-problem only considers $0.22 \%$ of the total number of constraints, similar savings are found in the storage memory of the Jacobian. If we assume double-precision, the memory storage requirement of the Jacobian of the complete set of constraints is estimated as $\sim 123 \mathrm{Mb}$, whereas the largest Jacobian in the working-set approach requires only $\sim 0.3 \mathrm{Mb}$.

Another important motivation of the proposed approach is to reduce the computational costs. Next, we compare CPU times. Since we implemented the function evaluations in Matlab 2017a, while IPOPT (Wächter and Biegler, 2006) is in $\mathrm{C}++$, Table 2 lists the CPU times of the function evaluations and IPOPT separately. The table shows that, for this particular example, the working-set approach was 164.8 times faster in IPOPT than when considering all constraints. Even though the total number of iterations is more when considering the working-set approach, the computational time in the function evaluations is 1.5 times less. The main savings are therefore in the time spent in the optimizer, which was expected since in both methods the function evaluations are dominated by solving the systems of equations in (1) and (10). The computational advantage of the working-set approach in the function evaluations is expected to be larger for problems with many load cases. The reason is that when there are many load cases, load cases for which no constraint is considered critical can be excluded from the analyses and sensitivity analysis when solving the sub-problem. In this case, the structure is subjected to only two load cases which were both deemed critical after the first sub-problem.

Figure 6 shows for every sub-problem the number of (a) constraints in the working set, (b) the number of critical loads, and (c) the number of violated constraints after solving each sub-problem. The number of constraints added after each sub-problem $k$ will depend on the constant $\epsilon$ in (3), which was chosen as $\epsilon=10^{-3}$. The effect of this parameter is discussed in the next example of the 25-bar frame structure. In Figure $6 \mathrm{~b}$ it can be seen that the number of critical loads reaches its maximum already after the second sub-problem. Figure $6 \mathrm{c}$ shows the number of violated constraints after each sub-problem on a logarithmic $y$-axis. The number of violated constraints decreases almost linear on the logarithmic axis; i.e. the number of violated constraints decreases exponentially. Notice that the number of violated constraints is zero after convergence of the seventh sub-problem; this value cannot be displayed on the logarithmic scale. 


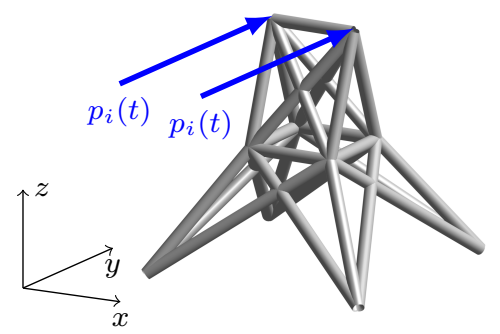

(a) Load cases I.

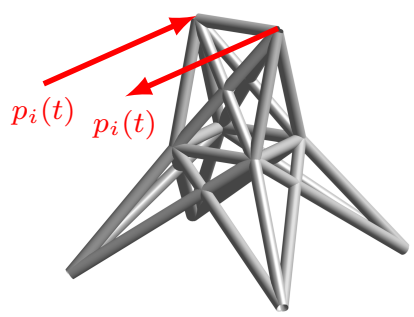

(b) Load cases II.

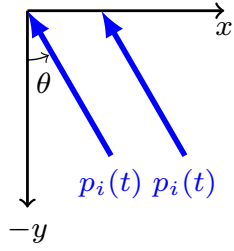

(c) Load orientation.

Fig. 7: Two load cases (a) and (b) on the 25-bar frame structure. Each load case is considered for 400s and applied under 18 different angles in the horizontal plane (c). The definition of the load cases is given in Table 3 .

Table 3: Load case definition for the 25-bar frame.

\begin{tabular}{lccccccccc}
\hline \multicolumn{1}{l}{ The loads are defined as } \\
\multicolumn{1}{c}{$p_{i}=\bar{p}_{i} \phi_{i}(t)$, where $\phi_{i}(t)=a_{0}+\sum_{i=1}^{3} a_{i} \sin \left(2 \pi f_{i} t\right)}$. \\
\hline $\begin{array}{l}\text { Load cases }\left(p_{i}(t)\right) \\
{[\mathrm{kN}]}\end{array}$ & $\begin{array}{c}\bar{p}_{i} \\
{[\mathrm{kN}]}\end{array}$ & $a_{0}$ & $a_{1}$ & $a_{2}$ & $a_{3}$ & $f_{1}$ & $f_{2}$ & $f_{3}$ \\
& & & & & & {$[\mathrm{~Hz}]$} & {$[\mathrm{Hz}]$} & {$[\mathrm{Hz}]$} \\
\hline $\mathrm{Ia}$ & 1500 & 0.40 & 0.05 & 0.25 & 0.30 & 3 & 0.80 & 0.009 \\
$\mathrm{Ib}$ & 1500 & 0.55 & 0.20 & 0.15 & 0.10 & 7 & 0.30 & 0.006 \\
$\mathrm{IIa}$ & 1500 & 0.80 & 0.10 & 0.05 & 0.05 & 5 & 0.20 & 0.003 \\
$\mathrm{IIb}$ & 1500 & 0.50 & 0.05 & 0.30 & 0.15 & 8 & 1.20 & 0.001 \\
\hline
\end{tabular}

\subsection{Example 2: A 25-bar frame structure}

Next, we discuss the effect of the user-defined parameter $\epsilon$ in the definition of the critical constraints (3). The effect was studied on the 25-bar frame structure shown in Figure 2b. The structure is fixed to the ground by constraining the degrees of freedom associated with the blue nodes. The number of free degrees of freedom is thus 36. The structure is subjected to two classes of time-dependent load cases: class I) load cases that produce an overturning moment as shown in Figure 7a, and class II) load cases that produce a torsional moment shown in Figure 7b. For each load class, two different load vectors are defined in the Table 3 resulting in four different load cases. The number of load cases is increased further by varying the orientation of each load vector in the horizontal plane. The angle $\theta$ in Figure $7 \mathrm{c}$ represents the orientation of each load vector, where $\theta=0^{\circ}$ corresponds with the load cases shown in Figure 7a and Figure 7b. Each of the original four load cases is applied under the following 18 orientations: $\theta=\left[-90^{\circ},-80^{\circ}, \ldots, 80^{\circ}\right]$. Consequently, a total of 72 separate load cases is applied to the structure. All load cases are considered for $400 \mathrm{~s}$, where the dynamic response during the first $100 \mathrm{~s}$ is neglected during optimization.
The optimization problem is to minimize the mass of the structure subjected to time-dependent stress constraints that have to hold at all relevant time steps. We consider 12 normal stress points in each beam element, which results in 300 spatial stress points. The dynamic stress responses at each spatial point results in 15001 stress constraints per load case after time discretization (for $\Delta t=0.02 \mathrm{~s}$ ). For a total of 72 load cases, the total number of stress constraints in this problem is, therefore, more than 324 million (324021600).

We investigated the effect of $\epsilon$ on the performance of the working-set approach by optimizing the 25-bar frame for different values on the logarithmic scale. We chose the following values: $\epsilon=a^{0} \underline{\epsilon}, a^{1} \underline{\epsilon}, \ldots, a^{14} \underline{\epsilon}$, where $a=\sqrt{10}$ and $\underline{\epsilon}=10^{-8}$. For each value of $\epsilon$, the mass of 25-bar frame was minimized subject to stress constraints. The parameter settings used during optimization are listed in Table 5. The initial design has a mass of $m=3966.25 \mathrm{~kg}$ and the maximum stress is $\sigma^{\max }=$ 309.8939 MPa.

For all values of $\epsilon$, the working-set approach converged for this particular problem to the same optimized design. Notice that this behavior of the algorithm is not guaranteed since the optimization problem is nonconvex and could have multiple local optima. Figure 8 shows the optimized design and its diameter values. The 


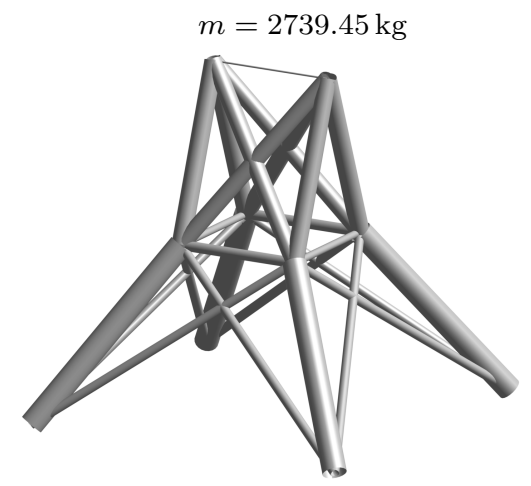

$\begin{aligned} D_{1} & =0.0100 & D_{9} & =0.1077 \\ D_{2} & =0.0971 & D_{10} & =0.0441 \\ D_{3} & =0.0994 & D_{11} & =0.0441 \\ D_{4} & =0.0994 & D_{12} & =0.0605 \\ D_{5} & =0.0971 & D_{13} & =0.0605 \\ D_{6} & =0.1077 & D_{14} & =0.0529 \\ D_{7} & =0.1032 & D_{15} & =0.0530 \\ D_{8} & =0.1032 & D_{16} & =0.0530\end{aligned}$

$D_{17}=0.0529$
$D_{18}=0.0536$
$D_{19}=0.0527$
$D_{20}=0.0527$
$D_{21}=0.0536$
$D_{22}=0.1398$
$D_{23}=0.1403$
$D_{24}=0.1398$
$D_{25}=0.1403$

Fig. 8: Optimized design of the 25-bar frame structure. In the figure, the diameters are scaled up a factor 5.

mass of the structure is $2739.45 \mathrm{~kg}$, which is $\sim 30.9 \%$ lower than the mass of the initial design. The maximum absolute stress value is on six decimals precision equal to the allowable stress of $240 \mathrm{MPa}$ and is $\sim 22.6 \%$ lower than the maximum absolute stress from the initial design. Seven out of the 72 load cases are active at the optimized design, i.e. at least one of the stress constraints associated with that load case is active. Figure 9 shows the maximum absolute value per load case for both the initial and optimized design. The red dashed line represents the allowable stress value. The number of active constraints for the optimized design is 18 , which means that for some of the seven active load cases more than one constraint is active. Notice that the number of active constraints is of the same order as the number of

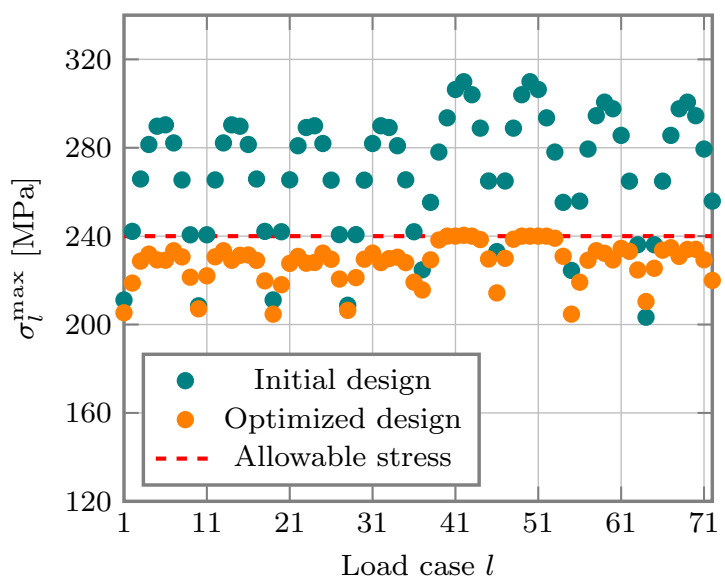

Fig. 9: Maximum absolute value of the normal stress $\sigma_{l}^{\max }$ for each load case $l$ for both the initial and optimized design. The maximum absolute stress is defined as $\sigma_{l}^{\max }=\max _{j, t}\left(\left|\sigma_{j t l}\right|\right)$ for each $l$. design variables (25), which confirms that only a few constraints are design-driving.

Although all optimization problems converged to the same optimized design for this particular problem, the parameter $\epsilon$ has an influence on the performance of the algorithm. Figure 10a shows a log-log plot with the number of constraints in the working set when the algorithm terminates, i.e., the working set of the final subproblem indicated by $\bar{k}$. We observe that the number of constraints decreases rapidly for decreasing values of $\epsilon$ in the range $\epsilon \in\left[10^{-3}, 10^{-1}\right]$. For values of $\epsilon$ small than $10^{-3}$, the number of constraints in the working set is more or less constant, and contains fewer than 100 out of the more than 324 million constraints of the original problem; i.e. only $\sim 3.1 \times 10^{-5} \%$ of the total number of constraints in the original problem was considered in the working-set approach. The same reduction is obtained in the required memory to store the dense Jacobian. If we assume that the largest working set contains 100 constraints (for a large range of $\epsilon$ it is even less) storing the Jacobian requires an estimated memory of $\sim 0.02 \mathrm{Mb}$ (assuming double-precision). Storing the Jacobian of the complete set of constraints requires $\sim 64804 \mathrm{Mb}$.

Figure 10b shows the critical loads at termination of the algorithm. The critical loads are the loads associated with the stress constraints in the working set, i.e.

$\mathcal{L}^{\bar{k}}=\left\{l \mid \exists(j, t, l) \in \mathcal{W}^{\bar{k}}\right\}$.

In each sub-problem, the stress constraint sensitivities are only computed for the set of critical loads. Consequently, this reduces the memory requirements and computational costs of the design sensitivity analysis. The number of critical load cases depends on the constraints in the working set, and the dependence of $\epsilon$ 


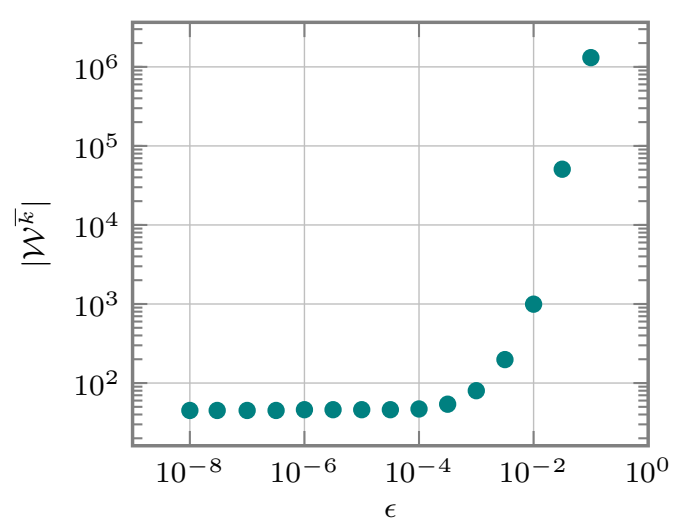

(a) Number of constraints in the final working set.

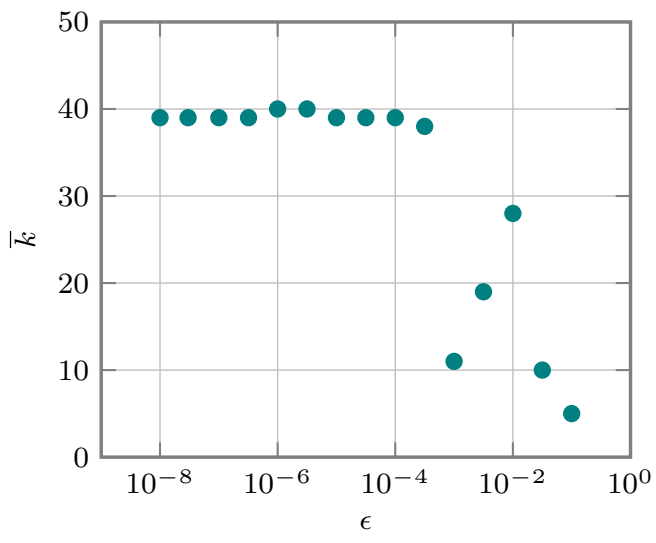

(c) Number of sub-problems.

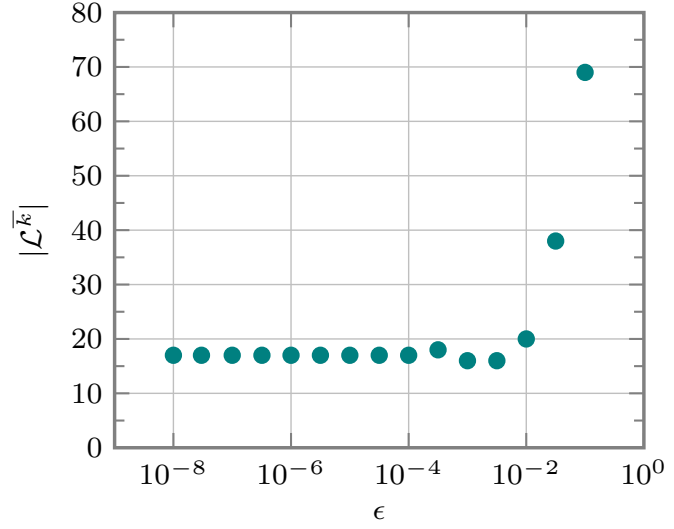

(b) Number of critical loads.

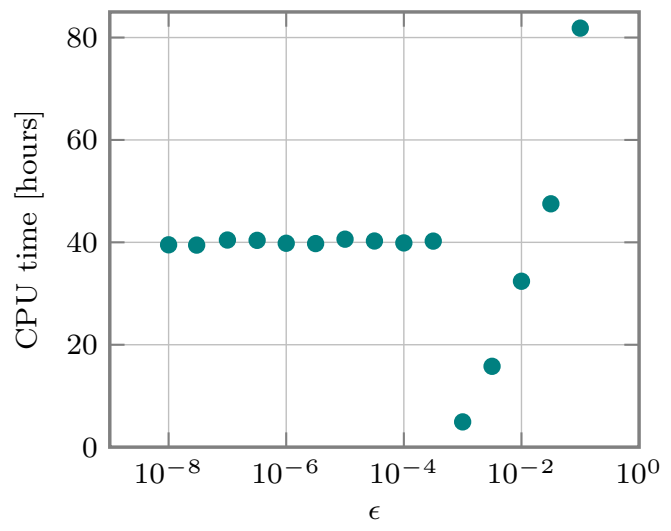

(d) CPU time.

Fig. 10: Results for different values of the parameter $\epsilon=\left\{a^{0}, a^{1}, \ldots, a^{14}\right\} \times \epsilon_{\min }$, where $a=\sqrt{10}$ and $\epsilon_{\min }=10^{-8}$. Here, $\bar{k}$ denotes the total number of sub-problems (outer iterates) that it took the algorithm to converge. (a) shows the number of constraints in the working set $\left|\mathcal{W}^{\bar{k}}\right|$ of the final sub-problem $\bar{k}$, (b) shows the number of critical load cases $\left|\mathcal{L}^{\bar{k}}\right|$, (c) the total number of sub-problems before the working-set algorithm terminates, and (d) the CPU time that it took to solve each problem instance.

follows a similar trend as the number of constraints in the working set versus $\epsilon$.

Figure 10c shows the total number of sub-problems before the algorithm terminated. We observe that the number of sub-problems is more or less constant in the range $\epsilon \in\left[10^{-8}, 10^{-4}\right]$. This behavior is similar to the number of constraints in the working set, which indicates that for this range of values the same constraints were identified as critical for this particular problem. We observe that the number of sub-problems fluctuates for relatively large values of the parameter (larger than $\left.10^{-4}\right)$. As $\epsilon$ becomes smaller, a smaller subset of critical constraints is identified, and therefore, more (but computationally cheaper) sub-problems need to be solved to identify all critical constraints. As $\epsilon$ becomes larger the sub-problem approximates the original problem, and therefore, if $\epsilon$ is close enough to one, the number of sub-problems should eventually converge to one, since for $\epsilon$ is equal to one all constraints of the original problem are included in the working set.

Figure 10d shows the total CPU time for each problem instance. The most efficient CPU times were found in the range $\epsilon \in\left[10^{-8}, 10^{-2}\right.$ ) for which the working set contains less than 1000 constraints. For this particular problem, $\epsilon=10^{-3}$ was most efficient in terms of CPU time. However, our numerical experience on other problems suggests that choosing a value $\epsilon \in\left[10^{-8}, 10^{-4}\right]$ generally gives the best trade-off in terms of CPU time versus the number of constraints in the working set and the associated memory requirement. For larger values of $\epsilon$, the number of constraints included in the working set grows exponentially, which lead to increased CPU times due to the increased computational expenses for the sensitivity analysis (due to the increase in critical 
loads) and the number of constraints considered in the optimizer.

\section{Advantages and limitations}

The main advantage of the working-set approach is that it greatly reduces the memory requirements and computational expenses for solving optimization problems with many constraints. The computational expenses are reduced in the (sensitivity) analysis and in the optimization solver. In particular, for problems with many load cases, the computational expenses of the sensitivity analysis can be greatly reduced by identifying the critical load cases; i.e. the load cases for which at least one constraints is design-driving.

An additional advantage of the proposed approach is its easy implementation since it does not require changes to the optimization method itself. Therefore, it works with any type of general purpose optimization solver for nonlinear optimization including SQP (e.g., Boggs and Tolle, 1995; Boggs and Tolle, 2000) and interior point methods (e.g., Forsgren, Gill, et al., 2002).

It should be noted that the approach can only be expected to work when the number of active constraints is expected to be relatively modest. This should be the case if the number of design variables is also modest (say in the order of hundreds). A disadvantage of the approach is its parameter-dependency on the critical parameter $\epsilon$. However, our numerical experiments indicate that the algorithm performs well and similar results are obtained for a large range of this parameter.

\section{Conclusions}

A working-set approach with advantageous theoretical properties has been proposed for sizing optimization problems of structures subjected to dynamic loads with relatively few variables but many constraints. The method is applied to structural optimization of frame structures subject to many loads and with many stress constraints. The numerical experiments demonstrate that for the considered sizing optimization problems a large number of time-dependent stress constraints can be handled effectively by solving a sequence of relaxed subproblem instead of the complete problem. Each subproblem considers only the constraints in the working set which includes the currently most critical constraints. The working set is updated after each problem is solved and is always ensured to contain the previous working set, i.e., the size of the working set increases until the algorithm terminates. The algorithm is robust and is easily implemented on top of existing off-theshelf numerical optimization methods for nonlinearly constrained problems. Future research will be directed towards efficient means also to reduce the size of the working set without compromising the theoretical properties.

It was found that for the intended application only a very few constraints are design-driving. This observation supports the hypothesis that structural optimization problems with few design variables also have few active constraints. Future research is focused on how to solve structural optimization problems with large numbers of design variables combined with a large number of constraints, such as topology optimization of framestructures subjected to dynamic loads.

Acknowledgements This research is part of the project ABYSS - Advancing BeYond Shallow waterS - Optimal design of offshore wind turbine support structures (www.abyss.dk). This project is sponsored by the Danish Council for Strategic Research, Grant no. 1305-00020B. This support is gratefully acknowledged.

\section{A Problem data and solver settings}


Table 4: Element connectivity. The nodal coordinates for both design problems can be seen in Figure 2. Each element is defined by three nodes $(i, j, k)$ as shown in Figure 1.

\begin{tabular}{|c|c|c|c|c|c|c|}
\hline \multirow[b]{2}{*}{ Element } & \multicolumn{3}{|c|}{ 16-bar frame } & \multicolumn{3}{|c|}{ 25-bar frame } \\
\hline & $i$ & $j$ & $k$ & $i$ & $j$ & $k$ \\
\hline 1 & 1 & 5 & 4 & 1 & 2 & 4 \\
\hline 2 & 2 & 6 & 1 & 4 & 1 & 2 \\
\hline 3 & 3 & 7 & 2 & 2 & 3 & 1 \\
\hline 4 & 4 & 8 & 3 & 5 & 1 & 6 \\
\hline 5 & 5 & 6 & 9 & 6 & 2 & 1 \\
\hline 6 & 6 & 7 & 10 & 2 & 4 & 3 \\
\hline 7 & 7 & 8 & 11 & 5 & 2 & 1 \\
\hline 8 & 8 & 5 & 12 & 3 & 1 & 2 \\
\hline 9 & 5 & 9 & 8 & 1 & 6 & 5 \\
\hline 10 & 6 & 10 & 5 & 6 & 3 & 10 \\
\hline 11 & 7 & 11 & 6 & 4 & 5 & 8 \\
\hline 12 & 8 & 12 & 7 & 4 & 3 & 1 \\
\hline 13 & 9 & 10 & 12 & 6 & 5 & 2 \\
\hline 14 & 10 & 11 & 9 & 3 & 10 & 6 \\
\hline 15 & 11 & 12 & 10 & 7 & 6 & 3 \\
\hline 16 & 12 & 9 & 11 & 9 & 4 & 5 \\
\hline 17 & - & - & - & 8 & 5 & 9 \\
\hline 18 & - & - & - & 4 & 7 & 3 \\
\hline 19 & - & - & - & 8 & 3 & 4 \\
\hline 20 & - & - & - & 10 & 5 & 6 \\
\hline 21 & - & - & - & 9 & 6 & 10 \\
\hline 22 & - & - & - & 6 & 10 & 9 \\
\hline 23 & - & - & - & 7 & 3 & 4 \\
\hline 24 & - & - & - & 4 & 8 & 7 \\
\hline 25 & - & - & - & 9 & 5 & 6 \\
\hline
\end{tabular}


Table 5: General settings

\begin{tabular}{ll}
\hline Option & Setting / Value \\
\hline & \multicolumn{1}{c}{ Model } \\
\hline Beam element & Euler-Bernoulli \\
Cross-section & Solid circular \\
Allowable stress & $\sigma_{\text {lim }}=240 \mathrm{MPa}$ \\
Young's Modulus & $E=68.9 \mathrm{GPa}$ \\
Poisson's ratio & $\nu=0.33$ \\
Density & $2770 \mathrm{~kg} \mathrm{~m}^{-3}$ \\
Rayleigh damping parameters & $\alpha=0.607, \beta=0.00064$ \\
Time discretization & Newmark average acceleration, time step $\Delta t=0.02 \mathrm{~s}$ \\
\hline & Problem formulation \\
\hline Minimum diameter $(\underline{\mathbf{x}})$ & $0.01 \mathrm{~m}$ \\
Maximum diameter $(\overline{\mathbf{x}})$ & $0.20 \mathrm{~m}$ \\
Initial diameters $\left(\mathbf{x}_{0}\right)$ & $(\underline{\mathbf{x}}+\overline{\mathbf{x}}) / 2=0.105 \mathrm{~m}$ \\
\hline \multicolumn{1}{c}{ Non-default parameters } & optimizer: ${ }^{*}$ IPOPT (Wächter and Biegler, 2006) \\
\hline mu_strategy & 'adaptive' \\
nlp_scaling_method & 'none' \\
hessian_approximation & 'limited-memory' (BFGS-approximation) \\
obj_scaling_factor & $1 \mathrm{e}-3$ \\
tol & $1 \mathrm{e}-6$ \\
constr_viol_tol & $1 \mathrm{e}-6$ \\
\hline
\end{tabular}

* A description of the IPOPT options can be found in (Options reference 2017). 


\section{References}

Arora, J. S., Chahande, A. I., and Paeng, J. K. (1991). "Multiplier methods for engineering optimization". In: International Journal for Numerical Methods in Engineering 32.7, pp. 1485-1525.

Bartlett, R. A. and Biegler, L. T. (2006). "QPSchur: large-scale and structured convex quadratic programming". In: Optimization and Engineering 7.1, pp. 532. A dual, active-set, Schur-complement method for

Forsgren, A., Gill, P., and Wright, M. (2002). "Interior methods for nonlinear optimization". In: SIAM Review 44.4, pp. 525-597.

Forsgren, A. and Murray, W. (1997). "Newton Methods For Large-Scale Linear Inequality-Constrained Minimization". In: SIAM Journal on Optimization 7.1, pp. $162-176$.

Fox, R. L. and Kapoor, M. P. (1970). "Structural optimization in the dynamics response regime - A computational approach". In: AIAA Journal 8.10, pp. 17981804.

\section{Programming". In: Acta numerica 4, pp. 1-51.}

Boggs, P. and Tolle, J. (2000). "Sequential quadratic programming for large-scale nonlinear optimization". In: Journal of Computational and Applied Mathematics 124.1-2, pp. 123-137.

Boland, N. L. (1996). "A dual-active-set algorithm for positive semi-definite quadratic programming". In: Mathematical Programming 78.1, pp. 1-27.

Bruggi, M. and Duysinx, P. (2012). "Topology optimization for minimum weight with compliance and stress constraints". In: Structural and Multidisciplinary Optimization 46.3, pp. 369-384.

Chahande, A. I. and Arora, J. S. (1994). "Optimization of large structures subjected to dynamic loads with the multiplier method". In: International Journal for Numerical Methods in Engineering 37.3, pp. 413430.

Choi, K. K. and Kim, N.-H. (2006). Structural sensitivity analysis and optimization 1: linear systems. New York: Springer-Verlag.

Choi, W. and Park, G. (2002). "Structural optimization using equivalent static loads at all time intervals". In: Computer Methods in Applied Mechanics and Engineering 191, pp. 2077-2094.

Cook, R. D. et al. (2002). Concepts and Applications of Finite Element Analysis, 4th Edition. New York: Wiley.

Duysinx, P. and Bendsøe, M. P. (1998). "Topology optimization of continuum structures with local stress constraints". eng. In: International Journal for $\mathrm{Nu}$ merical Methods in Engineering 43.8, pp. 1453-1478.

Duysinx, P. and Sigmund, O. (1998). "New developments in handling stress constraints in optimal material distribution". In: Multidisciplinary Analysis Optimization Conferences. American Institute of Aeronautics and Astronautics.

Feng, T.-T., Arora, J. S., and Haug, E. J. (1977). "Optimal structural design under dynamic loads". In: International Journal for Numerical Methods in Engineering 11.1, pp. 39-52. the integrated approach to structural synthesis." In: Journal of Spacecraft and Rockets 3.6, pp. 858-866.

Gill, P. E., Murray, W., and Saunders, M. A. (2005). "SNOPT: An SQP Algorithm for Large-Scale Constrained Optimization". In: SIAM Review 47.1, pp. 99131.

Goldfarb, D. and Idnani, A. (1983). "A numerically stable dual method for solving strictly convex quadratic programs". In: Mathematical Programming 27.1, pp. 133.

Grandhi, R. V., Haftka, R. T., and Watson, L. T. (1986). "Design-oriented identification of critical times in transient response". In: AIAA Journal 24.4, pp. 649656.

Greene, W. H. and Haftka, R. T. (1991). "Computational aspects of sensitivity calculations in linear transient structural analysis". In: Structural Optimization 3.3, pp. 176-201.

Haftka, R. T. (1975). "Parametric Constraints with Application to Optimization for Flutter Using a Continuous Flutter Constraint". In: AIAA Journal 13.4, pp. 471-475.

Haftka, R. T. and Shore, C. P. (1979). "Approximation methods for combined thermal/structural design". In: NASA Technical Paper 1428. Accessed: 201801-14.

Hestenes, M. R. (1969). "Multiplier and gradient methods". In: Journal of Optimization Theory and Applications 4.5, pp. 303-320.

Hsieh, C. and Arora, J. (1984). "Design sensitivity analysis and optimization of dynamic response". In: Computer Methods in Applied Mechanics and Engineering 43.2, pp. 195-219.

Options reference (2017). https ://www . coin-or.org/ Ipopt / documentation / node40 . html. Accessed: 2017-11-01.

Jensen, J. S., Nakshatrala, P. B., and Tortorelli, D. A. (2014). "On the consistency of adjoint sensitivity analysis for structural optimization of linear dynamic problems". In: Structural and Multidisciplinary Optimization 49.5, pp. 831-837. 
Kang, B.-S., Park, G.-J., and Arora, J. S. (2006). "A review of optimization of structures subjected to transient loads". In: Structural and Multidisciplinary Optimization 31.2, pp. 81-95.

Keulen, F. van, Haftka, R., and Kim, N. (2005). "Review of options for structural design sensitivity analysis. Part 1: Linear systems". In: Computer Methods in Applied Mechanics and Engineering 194.30-33, pp. 3213-3243.

Kim, M. and Choi, D.-H. (1998). "Min-max dynamic response optimization of mechanical systems using approximate augmented Lagrangian". In: International Journal for Numerical Methods in Engineering 43.3, pp. 549-564.

Kim, M. and Choi, D. (2000). "A new penalty parameter update rule in the augmented lagrange multiplier method for dynamic response optimization". In: KSME International Journal 14.10, pp. 11221130 .

Kreisselmeier, G. and Steinhauser, R. (1979). "Systematic Control Design by Optimizing a Vector Performance Index". In: IFAC Proceedings Volumes 12.7. IFAC Symposium on computer Aided Design of Control Systems, Zurich, Switzerland, 29-31 August, pp. 117.

Luenberger, D. G. and Ye, Y. (2008). Linear and Nonlinear Programming. 3rd. New York: Springer.

Murtagh, B. and Saunders, M. (1978). "Large-scale linearly constrained optimization". In: Mathematical Programming 14.1, pp. 41-72.

Muskulus, M. and Schafhirt, S. (2014). "Design optimization of wind turbine support structures-a review". In: Journal of Ocean and Wind Energy 1.1, pp. $12-22$.

Paeng, J. K. and Arora, J. S. (1989). "Dynamic Response Optimization of Mechanical Systems With Multiplier Methods". In: Journal of Mechanisms, Transmissions, and Automation in Design 111.1, pp. $73-80$.

Park, G. (2011). "Technical overview of the equivalent static loads method for non-linear static response structural optimization". In: Structural and Multidisciplinary Optimization 43.3, pp. 319-337.

Park, G. and Kang, B. (2003). "Validation of a structural optimization algorithm transforming dynamic loads into equivalent static loads". In: Journal of Optimization Theory and Applications 118.1, pp. 191200.

Powell, M. J. (1969). "A method for nonlinear constraints in minimization problems". In: Optimization. Ed. by R. Fletcher. New York: Academic Press, pp. 283-298.
Przemieniecki, J. S. (1985). Theory of matrix structural analysis. Courier Corporation.

Schmit, L. A. J. and Thornton, W. A. (1968). The structural synthesis of an ablating thermostructural panel. Tech. rep. NASA-CR-1215. Accessed: 201801-14. NASA.

Stolpe, M. (2014). "On the equivalent static loads approach for dynamic response structural optimization". In: Structural and Multidisciplinary Optimization 50.6, pp. 921-926.

Stolpe, M., Verbart, A., and Rojas-Labanda, S. (2017). "The equivalent static loads method for structural optimization does not in general generate optimal designs". In: Structural and Multidisciplinary Optimization.

Svanberg, K. (1987). "The method of moving asymptotesa new method for structural optimization". In: International journal for numerical methods in engineering 24.2, pp. 359-373.

Svanberg, K. (2002). "A class of globally convergent optimization methods based on conservative convex separable approximations". In: SIAM journal on optimization 12.2 , pp. $555-573$.

113Verbart, A. (2015). "Topology Optimization with Stress Constraints". PhD thesis. Delft University of Technology.

Wächter, A. and Biegler, L. T. (2006). "On the implementation of an interior-point filter line-search algorithm for large-scale nonlinear programming". In: Mathematical Programming 106.1, pp. 25-57.

Yang, R. J. and Chen, C. J. (1996). "Stress-based topology optimization". In: Structural optimization 12.2 , pp. $98-105$. 